\title{
High early postnatal weight gain linked to adult metabolic syndrome
}

A new study ascribes the known association between small birth size and development of the metabolic syndrome later in life to accelerated early postnatal weight gain. In the Journal of Clinical Endocrinology and Metabolism, Gerthe Kerkhof and colleagues from the Erasmus

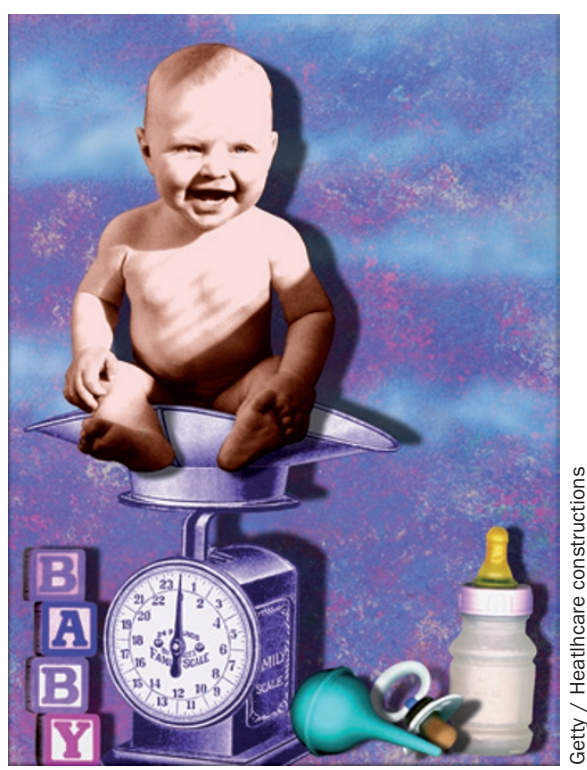

Medical Centre, the Netherlands, report that high weight gain relative to length in the first 3 months after birth is an important indicator of the metabolic syndrome in adulthood.

In a cohort of 280 healthy individuals aged 18-24 years who were enrolled in the PROGRAM study, Kerkhof and colleagues calculated SD scores (SDSs) for birth weight; weight and length at 3, 6, 9, and 12 months after birth; and insulinlike growth factor I (IGF-I) serum levels. The researchers then investigated the association of birth weight SDS, gain in weight for length SDS, and IGF-I SDS with the prevalence of the metabolic syndrome and related factors including C-reactive protein (CRP) levels, insulin sensitivity and cholesterol levels in adulthood.

An increase of one SDS in weight relative to length in the first 3 months of life was associated with a $34 \%$ increase in the number of metabolic syndrome components at age 21 , whereas no such associations were found for birth weight, weight gain during the other 3-month periods in the first year after birth, or adult IGF-I levels. Moreover, increased weight gain for length SDS in the first 3-month postnatal period was associated with increased prevalence of the metabolic syndrome, increased CRP levels, decreased HDL cholesterol levels and decreased insulin sensitivity in early adulthood, whereas low birth weight SDS was only associated with lower insulin sensitivity.

"These findings are in line with studies reporting an adverse effect of early-life weight gain on individual risk factors for cardiovascular disease and type 2 diabetes mellitus," explains Kerkhof. She calls for further research to determine the optimal target of early postnatal weight gain in infants, regardless of their birth weight, and to assess the effects of nutrition on the associations identified.

Iley Ozerlat

Original article Kerkhof, G. F. et al. Early origins of the metabolic syndrome: role of small size at birth, early postnatal weight gain, and adult IGF-I. J. Clin. Endocrinol. Metab. doi:10.1210/jc.2012-1426 